\title{
Authors' correction "Maintenance therapy with budesonide and formoterol in
chronic obstructive pulmonary disease" P.M. Calverley,
W. Boonsawat, Z. Cseke, N. Zhong, S. Peterson, H. Olsson.
Eur Respir J 2003; 22: 912-919.
}

It has been brought to the authors' attention by the safety group at AstraZeneca Lund Sweden that the manuscript "Maintenance therapy with budesonide and formoterol in chronic obstructive pulmonary disease" [1] contained inaccuracies in the safety data.

In the manuscript, one patient with a severe exacerbation of COPD was documented with a non-fatal outcome in the formoterol treatment group. The company, in the course of routine safety follow-up, later revealed that the outcome was fatal. The investigator reported the causality to study treatment as unlikely.

The safety data were correctly handled in line with regulatory authority reporting regulations. All authors have reviewed all additional information in light of the original data and agreed that these errors do not change the conclusions in the paper.

The finding initiated an extended quality check of the study safety data, which revealed a limited number of inconsistencies in the presentation of adverse events (AEs) (including serious AEs).

In light of this new information, the text in the Safety section (p. 917, beginning line 11, left-hand column) and table 5 (p. 917) should have read as follows.

"The lowest number of withdrawals was in the budesonide/formoterol group (table 1) and the lowest number of serious AEs other than deaths were in the budesonide/formoterol and placebo groups (64, 83, 84 and 67 in the budesonide/formoterol, budesonide, formoterol and placebo groups, respectively). The number of serious AEs related to COPD were 41, 40, 55 and 39 in the budesonide/formoterol, budesonide, formoterol and placebo groups, respectively. The number of deaths were 5, 6 , 14 and 5 in the budesonide/formoterol, budesonide, formoterol and placebo groups, respectively."

Page 917: table 5. The most frequently reported adverse events (AEs). In the B/F column, the correct percentage of patients with COPD reported as an AE should read 49 not 48.

Errors of this type can easily creep into the report of large multi-centre trials like this one, which involved investigators in many countries. The authors are grateful for this opportunity to present the corrected data. 